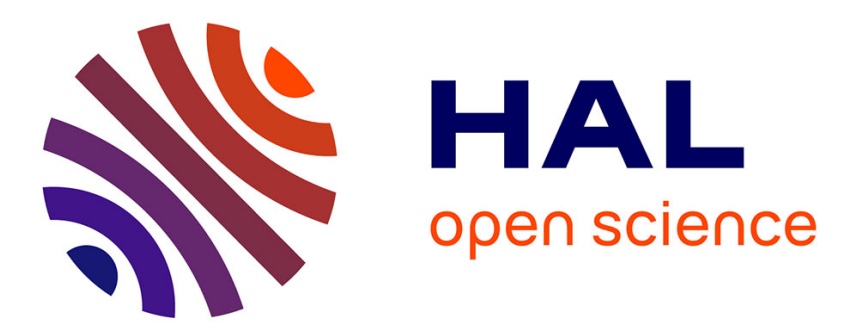

\title{
Pontin and reptin, two related ATPases with multiple roles in cancer.
}

\author{
Otmar Huber, Ludovic Ménard, Valérie Haurie, Alexandra Nicou, Danièle \\ Taras, Jean Rosenbaum
}

\section{- To cite this version:}

Otmar Huber, Ludovic Ménard, Valérie Haurie, Alexandra Nicou, Danièle Taras, et al.. Pontin and reptin, two related ATPases with multiple roles in cancer.: Pontin and Reptin in cancer. Cancer Research, 2008, 68 (17), pp.6873-6. 10.1158/0008-5472.CAN-08-0547 . inserm-00306362

\section{HAL Id: inserm-00306362 https://www.hal.inserm.fr/inserm-00306362}

Submitted on 3 Sep 2009

HAL is a multi-disciplinary open access archive for the deposit and dissemination of scientific research documents, whether they are published or not. The documents may come from teaching and research institutions in France or abroad, or from public or private research centers.
L'archive ouverte pluridisciplinaire HAL, est destinée au dépôt et à la diffusion de documents scientifiques de niveau recherche, publiés ou non, émanant des établissements d'enseignement et de recherche français ou étrangers, des laboratoires publics ou privés. 


\section{Pontin and Reptin, two related ATPases with multiple roles in cancer}

Otmar Huber ${ }^{1}$, Ludovic Ménard ${ }^{2}$, Valérie Haurie ${ }^{2}$, Alexandra Nicou ${ }^{2}$, Danièle Taras ${ }^{2}$, and Jean Rosenbaum ${ }^{2}$

${ }^{1}$ Charité-Universitätsmedizin Berlin, Campus Benjamin Franklin, Department of Laboratory Medicine and Pathobiochemistry, 12200 Berlin, Germany

${ }^{2}$ INSERM, U889; Université Victor Segalen Bordeaux 2, F-33076 Bordeaux, France

\section{Footnotes}

Financial support : supported by grants to JR from Institut National du Cancer, Association pour la Recherche sur le Cancer, Agence Nationale pour la Recherche sur le SIDA et les Hépatites Virales and Ligue Nationale Contre le Cancer, and by grants to OH from the Deutsche Forschungsgemeinschaft and the Sonnenfeld-Stiftung Reprint requests to Jean Rosenbaum : INSERM U889, Université Victor Segalen Bordeaux 2, 146 rue Léo Saignat, 33076 Bordeaux cedex, France ; e-mail : jean.rosenbaum@gref.u-bordeaux2.fr

Running title : Pontin and Reptin in cancer

Key words : ATPase, helicase, beta-catenin, c-myc, Tip60, telomerase, SUMO 


\begin{abstract}
Studies in model organisms or cultured human cells suggested potential implications in carcinogenesis for the AAA+ ATPases Pontin and Reptin. Both proteins are associated to several chromatin-remodeling complexes and have many functions, including transcriptional regulation, DNA damage repair and telomerase activity. They also interact with major oncogenic actors such as $\beta$-catenin and c-myc and regulate their oncogenic function. We only now begin to get insight into the role of Pontin and Reptin in human cancers.
\end{abstract}

\title{
Introduction
}

Pontin and Reptin are two related members of the AAA+ (ATPases associated with diverse cellular activities) superfamily sharing conserved Walker A and B motifs, arginine fingers and sensor domains. Since both proteins were identified independently by several groups, multiple names exist in the literature and data bases (RuvB11, Rvb1, Tip49a, NMP238, ECP54, TAP54 $\alpha$, TIH1 for Pontin, and RuvB12, Tip49b, ECP51, TAP54 $\alpha$, TIH2 for Reptin). Pontin and Reptin share limited homology to the bacterial helicase RuvB, which is essentially involved in the catalysis of Holliday junction branch migration. Whereas the functional importance of the ATPase activity of both proteins has been clearly established by the expression of proteins mutated in the Walker A or B motives, there is dispute whether they are indeed endowed with helicase activity. In the recent years, a number of studies have established that both proteins are essential for the viability and development of model organisms including $S$. cerevisiae, D. melanogaster, X. laevis or D. rerio.

Pontin and Reptin normally are coexpressed and frequently share common binding partners. Formation of homomeric and heteromeric interactions between Pontin and/or Reptin has been 
reported but heteromeric complex formation is preferred as analyzed by coimmunoprecipitation from lysates of transfected cells. In vitro, Pontin and Reptin form hexameric or double hexameric ring systems (1). Most evidence points to an association of Pontin and Reptin in the cell nucleus with several types of high molecular weight complexes involved in chromatin remodeling and/or transcriptional regulation such as in human the hINO80, Tip60, SRCAP and Uri1/Prefoldin complexes (1). It is however noteworthy that the multimeric status of Pontin and Reptin in the cytoplasm is not well defined. Some data even suggest that they are found in different subcellular locations in the course of cell division (2). The structural aspects of Pontin and Reptin and their functions in the regulation of transcription have been recently reviewed (1) and will thus not be addressed here in detail.

\section{Expression in cancer}

Until recently, there were no data regarding the expression of Pontin and Reptin in human cancer. We performed a differential proteomic analysis of a few cases of human HCC that were compared to the non-tumor surrounding liver (3). This revealed an overexpression of Reptin in the index cases that led us to study a further 96 cases of human HCC with real-time PCR. It turned out that Reptin transcription was increased in $75 \%$ of the cases (same for Pontin, unpublished data) (4) and that high levels of Reptin were correlated with a poor prognosis, independently of other prognosis variables. We have also investigated Pontin expression in a series of 34 colon cancer specimen and found it increased in more than $80 \%$ of the cases (5). The regulation of Reptin or Pontin expression has not been reported as such in other large series of tumors. However, re-analysis of microarray data available in the Oncomine database (http://www.oncomine.org) shows a deregulated expression of these proteins in several cancers such as those of bladder and in melanoma. 
The mechanisms inducing overexpression of Reptin and Pontin in tumors are unknown and may be tumor type-specific. The Reptin gene is located on chromosome 19q13.3, a region not commonly affected in HCC and it is thus unlikely that Reptin overexpression is consecutive to a gene amplification event. The same is true for the Pontin gene, which is located on chromosome 3q21. In contrast, Pontin is overexpressed in non-small cell lung cancer (6) where this locus is commonly amplified.

Unexpectedly, we detected strongly enhanced cytoplasmic expression of Reptin (4) and Pontin (unpublished) in liver and of Pontin in colon (5) cancer. All known functions of Pontin and Reptin are consistent with a nuclear localization, and both proteins indeed have been localized in the nucleus in many cell types from various organisms. On the other hand, both Reptin and Pontin can be found associated with the mitotic apparatus, although at different locations during mitosis, suggesting that they may play distinct roles during this process independently of their nuclear functions (2).

Because Pontin and Reptin expression appears increased in a large number of cancer types, these proteins may be of general interest for oncologists. The specific nuclear and non-nuclear functions of both proteins remains to be dissected and may elucidate important aspects regarding their roles in cancer cells.

\section{Interactions with mediators of carcinogenesis}

Numerous Pontin and Reptin interaction partners have known roles in cancer. The first hints suggesting a relationship between Pontin, Reptin and cancer came with the discovery that both proteins interacted with $\beta$-catenin $(7,8)$ and c-myc (9). Moreover, expression of both proteins is regulated by c-myc (9). Pontin appears to be required for the transforming effect of 
c-myc (9), the viral oncoprotein E1A (10), or ß-catenin (11). There are no similar data available for Reptin. On the other hand, in various assays, Reptin was found to antagonize the transcriptional effect of the TCF/LEF-1- $\beta$-catenin complex, whereas Pontin potentiated it (8). However, more recently it was shown in metastatic prostate cancer cells that Reptin, but not Pontin, was recruited on the promoter of the metastasis suppressor gene KAI-1 in a complex with $\beta$-catenin and was required for the repressing effect of $\beta$-catenin on the transcription of this gene, possibly contributing to enhanced invasive properties of tumor cells (12). The formation of this complex appears to depend on sumoylation of Reptin (13). Conversely, in non-metastatic prostate cancer cells, Pontin, but not Reptin, is recruited to the same promoter in complex with the Tip60 histone acetyltransferase, although Pontin is not required for the activating effect of Tip60 on transcription (12). On the other hand, Pontin turned out to be a co-factor for the transcriptional activation of androgen receptor target genes in a sumoylationdependent way (14).

Tip60 is a multifaceted protein, also involved in DNA damage repair, where it operates within a multi-protein complex containing both Pontin and Reptin (15). Their likely role in the response to DNA damage is in agreement with the recent observations that both Pontin and Reptin appear to be phosphorylated by ATM/ATR following DNA damage (16), and that Pontin is required for Tip60 activity after DNA damage (17).

We have also shown that Pontin and Reptin bind the tumor suppressor Hint1/PKC1 (histidine triad nucleotide-binding protein 1/protein kinase $\mathrm{C}$ inhibitor 1) (18). Hint1 binding can disrupt the Pontin-Reptin interaction, but it is not known whether Reptin or Pontin modulate Hint1 functions. Reptin, but not Pontin also interacts with the transcription factor ATF-2 (19), that can function either as a tumor susceptibility protein or a tumor suppressor according to the cell type. Reptin interferes with the transcriptional activity of ATF-2 and this may participate in its function. 
Thus, Pontin and Reptin interact with many established actors of carcinogenesis. Although they may appear to act antagonistically at the molecular level, they both are involved in events that favor tumor progression.

\section{Roles in cell viability/cell death}

As mentioned above, the absence of expression of either Pontin or Reptin impairs cell growth in several organisms. The presence of one protein cannot compensate for the deficiency of the other. Loss of function mutations in Walker domains have similar effects on growth as the absence of the protein, likely indicating a requirement for the ATPase activity. Conversely, overexpression of Pontin or Reptin in the Xenopus embryo leads to an increased proliferation, an effect that is independent of the function of Walker domains but is likely instead related to interactions with c-myc (20).

Although interactions with c-myc may be involved $(20,21)$, the mechanisms through which Pontin and Reptin regulate cell growth remain incompletely understood. Several data point to a role in the G1 part of the cell cycle. Indeed, loss of Reptin in yeast induces a blockage in G1 (22). This is coherent with the observation that both proteins are involved in transcriptional repression of $\mathrm{p} 21$, a repressor of the cyclin E/cdk2 complex required for the G1/S transition (20). In human HCC cells we have observed a concomitant block in G2/M after knock-down of Reptin (unpublished data). Intriguingly, abrogation of Pontin or Reptin expression has been shown to induce premature senescence, which may contribute to the observed effects on the cell cycle (23). This is in line with the very recent finding that Pontin and Reptin are required for the assembly and function of the telomerase complex (24). 
Besides their role in regulating cell proliferation, both Pontin and Reptin are involved in the regulation of apoptosis. We observed that siRNA-mediated knock-down of Reptin in human HCC cells led to spontaneous apoptotic cell death (4). Similar results were obtained in other tumor cell lines, either hepatic, or not (MCF-7, unpublished data). Conversely, transduction with a lentiviral vector coding Flag-tagged Reptin, that induced a moderate overexpression of Reptin, conferred an increased resistance to apoptotic cell death in HCC cells (4). Strikingly, Tyteca et al. found that siRNA-mediated knock-down of Reptin in the human osteosarcoma cell line U2OS had the opposite effect in decreasing UV-induced apoptosis (25). This discrepancy may be explained by the fact that UV-induced cell death is dependent of p53 activation, and Reptin acts as a partner in the Tip60 complex that functions as a co-activator of p53 (25). On the other hand, we have found that the spontaneous apoptosis following knock-down of Reptin in a variety of HCC cells occurred whether p53 was wild-type or mutated (unpublished data).

Pontin also appears to act as an anti-apoptotic factor. Indeed, a dominant-negative mutant of Pontin, devoid of ATPase activity, potentiates the apoptotic activity of c-myc and of E2F1 (10). We found that, similar to Reptin, the knock-down of Pontin in HCC cells led to spontaneous apoptosis ${ }^{1}$.

As mentioned above, Pontin and Reptin interact with Hint1, which triggers apoptosis through binding to and activating notably the Bax promoter (26). An attractive hypothesis is that the increased cytoplasmic levels of both proteins observed in some malignancies may sequester Hint1 in the cytoplasm preventing it from regulating pro-apoptotic gene expression.

Altogether, Pontin and Reptin favor cell proliferation and inhibit apoptosis, properties well in line with their supposed roles in cancer.

\footnotetext{
${ }^{1}$ Haurie V, Ménard L, Taras D and Rosenbaum J, manuscript in preparation
} 


\section{Pontin and Reptin as therapeutic targets?}

As mentioned above, decreased expression of either Pontin or Reptin results in reduced tumor cell growth and increased apoptosis in vitro (4) (and unpublished observations). We have also found that decreasing Reptin expression results in growth arrest of established tumors in xenograft experiments in mice ${ }^{2}$. Thus, these proteins may qualify as therapeutic targets. It is also interesting to note that Reptin and Pontin are endowed with an ATPase activity, shown to be required for most of their known functions, which could be targeted by specific inhibitors.

\section{Conclusion}

Pontin and Reptin remain intriguing proteins that, given their alleged functions, appear to be involved in carcinogenesis at a multitude of steps. Much remains to be understood about them, but one of the most challenging issues may be the understanding of their respective roles. Indeed, although they are most often found in the same complexes, this is not always the case (12). In addition, both proteins have been evolutionary conserved, suggesting that they have distinctive functions, which is supported by the finding that the deficiency of one can not be rescued by expression of the other (9). It is likely that both the nature of the multimeric complexes they form, and the partners they interact with, will dictate their function in a variety of cellular processes relevant for carcinogenesis (Fig. 1).

\footnotetext{
${ }^{2}$ Ménard L, Taras D, Nicou A, Haurie V and Rosenbaum J, manuscript in preparation.
} 


\section{References}

1. Gallant P. Control of transcription by Pontin and Reptin. Trends Cell Biol 2007;17: 187-92.

2. Sigala B, Edwards M, Puri T, Tsaneva IR. Relocalization of human chromatin remodeling cofactor TIP48 in mitosis. Exp Cell Res 2005;310: 357-69.

3. Blanc J, Lalanne C, Plomion C, et al. Proteomic analysis of differentially expressed proteins in hepatocellular carcinoma developed in patients with chronic viral hepatitis C. Proteomics 2005;5: 3778-89.

4. Rousseau B, Menard L, Haurie V, et al. Overexpression and role of the ATPase and putative DNA helicase RuvB-like 2 in human hepatocellular carcinoma. Hepatology 2007;46: 1108-18.

5. Lauscher JC, Loddenkemper C, Kosel L, et al. Increased pontin expression in human colorectal cancer tissue. Hum Pathol 2007;38: 978-85.

6. Dehan E, Ben-Dor A, Liao W, et al. Chromosomal aberrations and gene expression profiles in non-small cell lung cancer. Lung Cancer 2007;56: 175-84.

7. Bauer A, Huber O, Kemler R. Pontin52, an interaction partner of ß-catenin, binds to the TATA box binding protein. Proc Natl Acad Sci U S A 1998;95: 14787-92.

8. Bauer A, Chauvet S, Huber O, et al. Pontin52 and reptin52 function as antagonistic regulators of ß-catenin signalling activity. EMBO J 2000;19: 6121-30.

9. Wood MA, McMahon SB, Cole MD. An ATPase/helicase complex is an essential cofactor for oncogenic transformation by c-Myc. Mol Cell 2000;5: 321-30.

10. Dugan KA, Wood MA, Cole MD. TIP49, but not TRRAP, modulates c-Myc and E2F1 dependent apoptosis. Oncogene 2002;21: 5835-43. 
11. Feng Y, Lee N, Fearon ER. TIP49 regulates ß-catenin-mediated neoplastic transformation and T-cell factor target gene induction via effects on chromatin remodeling. Cancer Res 2003;63: 8726-34.

12. Kim JH, Kim B, Cai L, et al. Transcriptional regulation of a metastasis suppressor gene by Tip60 and ß-catenin complexes. Nature 2005;434: 921-6.

13. Kim JH, Choi HJ, Kim B, et al. Roles of sumoylation of a reptin chromatinremodelling complex in cancer metastasis. Nat Cell Biol 2006;8: 631-9.

14. Kim JH, Lee JM, Nam HJ, et al. SUMOylation of pontin chromatin-remodeling complex reveals a signal integration code in prostate cancer cells. Proc Natl Acad Sci U S A 2007;104: 20793-8.

15. Ikura T, Ogryzko VV, Grigoriev M, et al. Involvement of the TIP60 histone acetylase complex in DNA repair and apoptosis. Cell 2000;102: 463-73.

16. Matsuoka S, Ballif BA, Smogorzewska A, et al. ATM and ATR substrate analysis reveals extensive protein networks responsive to DNA damage. Science 2007;316: 1160-6.

17. Jha S, Shibata E, Dutta A. Human Rvb1/Tip49 is required for the histone acetyltransferase activity of Tip60/NuA4 and for the downregulation of phosphorylation on H2AX after DNA damage. Mol Cell Biol 2008;28: 2690-700.

18. Weiske J, Huber O. The histidine triad protein Hint1 interacts with Pontin and Reptin and inhibits TCF- ß-catenin-mediated transcription. J Cell Sci 2005;118: 3117-29.

19. Cho SG, Bhoumik A, Broday L, et al. TIP49b, a regulator of activating transcription factor 2 response to stress and DNA damage. Mol Cell Biol 2001;21: 8398-413.

20. Etard C, Gradl D, Kunz M, Eilers M, Wedlich D. Pontin and Reptin regulate cell proliferation in early Xenopus embryos in collaboration with c-Myc and Miz-1. Mech Dev 2005;122: 545-56. 
21. Bellosta P, Hulf T, Balla Diop S, et al. Myc interacts genetically with Tip48/Reptin and Tip49/Pontin to control growth and proliferation during Drosophila development. Proc Natl Acad Sci U S A 2005;102: 11799-804.

22. Lim CR, Kimata Y, Ohdate H, et al. The Saccharomyces cerevisiae RuvB-like protein, Tih2p, is required for cell cycle progression and RNA polymerase II-directed transcription. J Biol Chem 2000;275: 22409-17.

23. Chan HM, Narita M, Lowe SW, Livingston DM. The p400 E1A-associated protein is a novel component of the p53 --> p21 senescence pathway. Genes Dev 2005; 19: 196201.

24. Venteicher AS, Meng Z, Mason PJ, Veenstra TD, Artandi SE. Identification of ATPases pontin and reptin as telomerase components essential for holoenzyme assembly. Cell 2008;132: 945-57.

25. Tyteca S, Vandromme M, Legube G, Chevillard-Briet M, Trouche D. Tip60 and p400 are both required for UV-induced apoptosis but play antagonistic roles in cell cycle progression. EMBO J 2006;25: 1680-9.

26. Weiske J, Huber O. The histidine triad protein Hint1 triggers apoptosis independent of its enzymatic activity. J Biol Chem 2006;281: 27356-66. 


\section{Figure legends}

Figure 1: A cartoon depicting the context-dependent complexity of Pontin and Reptin

functions. Depending on their multimeric status (either monomers, homo-oligomers, hetero-oligomers) and their main interaction partners (within the yellow circle), they are involved in multiple biological processes (green circle). However, the detailed molecular mechanisms behind these activities have to be deciphered. 


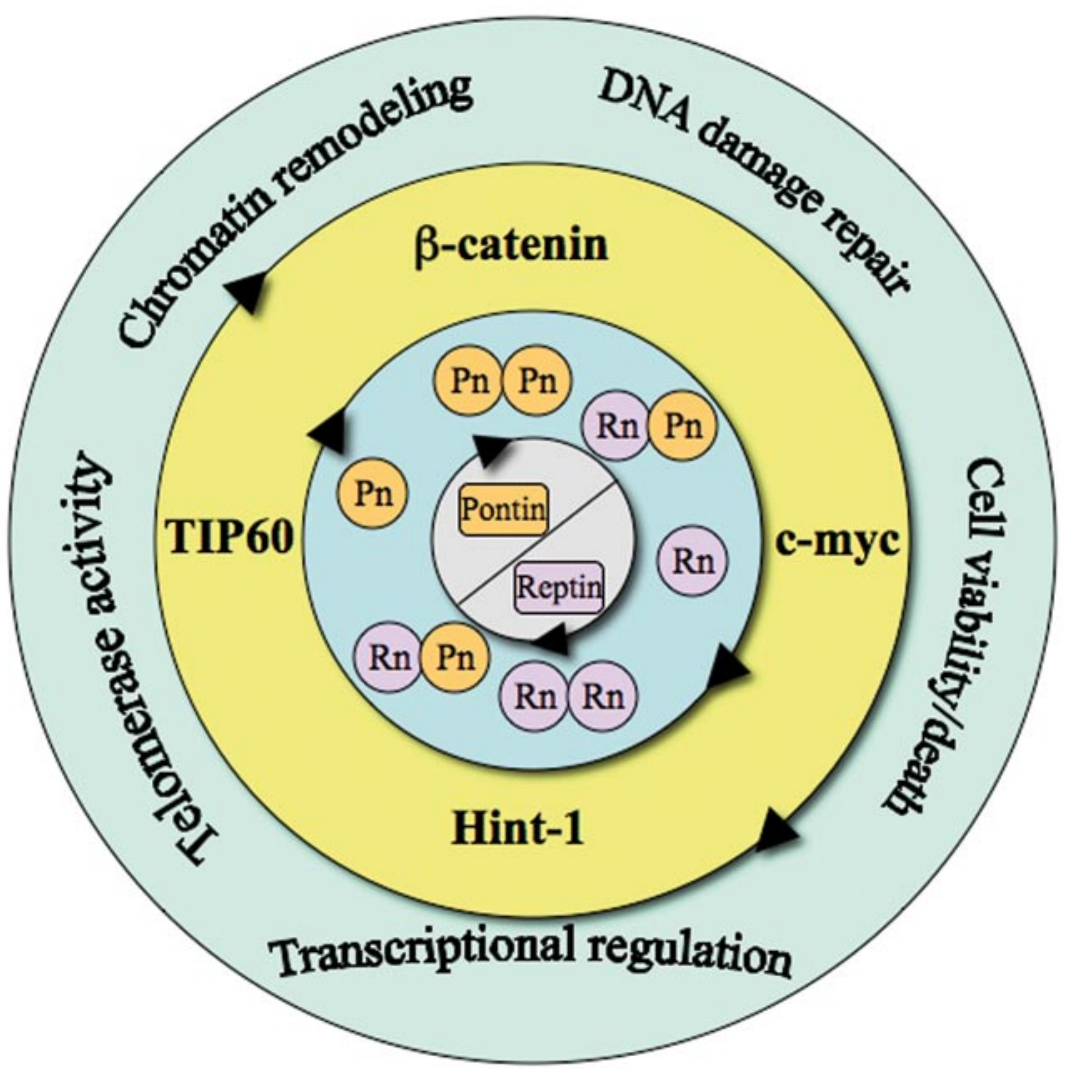

